\title{
O USO DO CHATBOT PARA A EXCELÊNCIA EM ATENDIMENTO
}

\author{
THE USE OF CHATBOT FOR EXCELLENCE IN SERVICE
}

\author{
Verônica Adelaide Lugli - veronicaad@hotmail.com \\ João de Lucca Filho - joaodelucca@terra.com.br
}

Faculdade de Tecnologia de Taquaritinga (Fatec) - Taquaritinga - São Paulo - Brasil

DOI: 10.31510/infa.v17i1.840

\begin{abstract}
RESUMO
A excelência em atendimento é um diferencial para empresas que necessitam de uma central de atendimento para que seus clientes possam entrar em contato sempre que necessário. Deste modo, a implantação de um chatbot permite obter melhores resultados e com atendimentos ágeis. Neste estudo são apresentados alguns referenciais teóricos que permitem o entendimento sobre chatbots, bem como aspectos importantes para sua evolução e humanização, aproximando-se cada vez mais do raciocínio e da linguagem humana. O objetivo deste artigo é mostrar que o uso dessa tecnologia é vantajoso para empresas que buscam a excelência em atendimento a clientes, diminuindo a sobrecarga de problemas levados aos operadores, sendo que muitas dúvidas e contratempos podem ser resolvidos pelo próprio chatbot, sem a necessidade de uma interação humana. Para demonstrar a eficiência inerente dessa inovação, é apresentado um estudo de caso com a empresa Grupo Oportunidade e seus resultados, que está utilizando a tecnologia de chatbot em sua central de atendimento, com foco na redução do tempo de espera para seus usuários serem atendidos, e apresentando soluções aos problemas de forma mais ágil. As conclusões deste estudo indicam que o chatbot é uma vantagem para as empresas, porém para atingir todos os novos objetivos ou metas propostas, observa-se a necessidade de um contínuo processo de novas melhorias.
\end{abstract}

Palavras-chave: Chatbot. Excelência em atendimento. Humanização. Inteligência Artificial.

\begin{abstract}
Service excellence is a differential for companies that need a call center so that their customers can contact whenever necessary. In this way, the implantation of a chatbot allows to obtain better results and with agile services. In this study are presented some theoretical references that allow the understanding of chatbots, with also relevant aspects for the evolution and humanization of chatbots, bringing the reasoning capacity and the current human language. This article shows that the use of chatbots is advantageous for companies seeking excellence in service to its customers. The chatbot possible to reduce the overhead of problems treated by operators, allows many doubts and setbacks can be solved by itself chatbot, without the need for human interaction. To demonstrate the inherent efficiency of this innovation, a case study of Grupo Oportunidade with its results is presented. The company is using the chatbot technology in its call center, the focus of the paradigm shift was to reduce the waiting time for
\end{abstract}


service of its members, and then present solutions to problems in a more agile way. The conclusions of this study indicate that the chatbot was advantageous for the company, but to achieve all the new objectives or goals proposed, there is a need for a continuous process of new improvements.

Keywords: Chatbot. Excellence in service. Humanization. Artificial intelligence.

\section{INTRODUÇÃO}

A Inteligência Artificial (IA) é uma área em contínua ebulição que tem sido muito discutida e vem apoiando o avanço tecnológico, além de permitir emular tanto a capacidade quanto alguns aspectos da inteligência humana.

Diante dessa ebulição em um processo de constante evolução, foi possível a criação de diversas tecnologias que, atualmente, estão cada vez mais presentes no cotidiano das pessoas e um exemplo disso é o uso de chatbot para atendimentos em geral.

Para uma empresa no cenário de relacionamentos da atualidade, é inviável oferecer atendimento 24 horas com atendente humano, porém, com o uso de tecnologia de assistentes virtuais é possível a criação de uma plataforma utilizando aprendizado de máquina que utiliza a processamento de linguagem natural para conversar com os clientes de uma empresa.

O objetivo deste trabalho é demonstrar que o uso da tecnologia de chatbot voltado para atendimento ao cliente, com excelência, é vantajoso para a empresa. E para comprovar foi realizada a aplicação de um caso real, com essa inovação para o atendimento ao cliente.

Neste trabalho é apresentado a fundamentação teórica, muito importante para o entendimento do chatbot, assim como a contextualização sobre Inteligência Artificial e a humanização das máquinas. Em seguida, é apresentado um estudo de caso do Grupo Oportunidade que está implantando a tecnologia chatbot para o atendimento ao cliente e as consequências que trouxe para a empresa. Logo após é mostrado o resultado de todo o estudo feito na empresa, seguindo as considerações finais e referências.

\section{INTELIGÊNCIA ARTIFICIAL}

Vários autores afirmam ser complexo definir Inteligência Artificial. Neste contexto, Rosa (2011, p.3) explica que a palavra artificial significa algo que é produzido pela ação do homem, e também, diz que definir a inteligência é algo complexo. Já Luger (1998, p.1) diz que 
a IA pode ser definida como um ramo da ciência da computação que se preocupa com a automação do comportamento inteligente.

Russell e Norving (2013) relacionam esses processos da IA por meio do ato de pensar e agir, sejam eles de forma humana ou racionalmente. Como apresentado na Ilustração 1, de um ponto de vista histórico existem quatro estratégias para tal definição, seja ela centrada em seres humanos, envolvendo uma ciência empírica e experimental, até a abordagem racional que se utiliza das teorias matemáticas e das engenharias.

\section{Ilustração 1 - Definições de Inteligência Artificial}

\begin{tabular}{|c|c|}
\hline Pensando como um Humano & Pensando Racionalmente \\
\hline $\begin{array}{l}\text { "O novo e interessante esforço para fazer os computadores } \\
\text { pensarem (...) máquinas com mentes, no sentido totale } \\
\text { literal." (Haugeland, 1985) } \\
\text { "[Automatização de] atividades que associamos ao } \\
\text { pensamento humano, atividades como a tomada de decisões, } \\
\text { a resolução de problemas, o aprendizado..."(Bellman, 1978) }\end{array}$ & $\begin{array}{l}\text { "O estudo das faculdades mentais pelo uso de modelos } \\
\text { computacionais." (Charniak e McDermott, 1985) } \\
\text { "O estudo das computações que tornam possível perceber, } \\
\text { raciocinar e agir." (Winston, 1992) }\end{array}$ \\
\hline Agindo como seres Humanos & Agindo Racionalmente \\
\hline $\begin{array}{l}\text { "A arte de criar máquinas que executam funções que exigem } \\
\text { inteligência quando executadas por pessoas."(Kurzweil, } \\
1990) \\
\text { "O estudo de como os computadores podem fazer tarefas } \\
\text { que hoje são melhor desempenhadas pelas pessoas."(Rich } \\
\text { and Knight, 1991) }\end{array}$ & $\begin{array}{l}\text { "Inteligência Computacional é o estudo do projeto de } \\
\text { agentes inteligentes." (Poole et al., 1998) } \\
\text { "AI... está relacionada a um desempenho inteligente de } \\
\text { artefatos."(Nilsson, 1998) }\end{array}$ \\
\hline
\end{tabular}

Fonte: Adaptado de Russell e Norving (2013)

O agir de forma humana consiste na teoria proposta por Alan Turing por volta de 1950, que projetou algumas capacidades que o computador precisa ter para ser de inteligência satisfatória, fazendo com que a máquina converse com um humano e este não descubra de quem vêm as respostas. Para isso, o sistema necessita de uma comunicação natural além de ser capaz de armazenar e usar informações obtidas, de poder pensar para responder às questões e tomar decisões (RUSSELL e NORVING, 2013).

Já o pensar humanamente utiliza uma ciência cognitiva reunindo modelos da IA e da psicologia, a fim de construir modelos computacionais precisos sobre o funcionamento da mente humana (RUSSELL e NORVING, 2013).

Quando se pensa racionalmente, a fim de buscar resultados e conclusões, de forma precisa e lógica, são utilizadas as leis do pensamento. Tal característica apresenta alguns problemas, tais como agir corretamente, da mesma forma que a máquina tem pensado, e o esgotamento dos recursos físicos da máquina (RUSSELL e NORVING, 2013).

Por fim, o agir racionalmente depende de um agente racional, ou seja, alguém capaz de fazer algo que pode ser uma máquina autônoma que conhece seu ambiente e aja sobre ele, buscando sempre as melhores soluções e caminhos (RUSSELL e NORVING, 2013). 
O objetivo da IA é, portanto, o desenvolvimento de algoritmos, soluções e paradigmas que faz as máquinas realizarem tarefas com a absorção de conhecimento, de forma equivalente ou até melhor que os seres humanos fazem atualmente (HAYKIN, 1999 apud SAGE, 1990).

Um bom sistema de IA deve procurar uma maneira de entender e aplicar soluções inteligentes para problemas através de um conhecimento adquirido e armazenado, além de planejar e apresentar habilidade de se comunicar, com a finalidade de buscar novas informações através da experiência (LUGER, 2009).

Algumas disciplinas como filosofia, matemática, economia, neurociência, psicologia, engenharia de computadores, teoria de controle, cibernética e linguística foram importantes para o desenvolvimento da IA, segundo Russell e Norving (2013), pois contribuíram com ideias e teorias para pontos de vistas e técnicas da IA, desde a descoberta de como o conhecimento é adquirido, a maneira que isso é computado e processado por um cérebro humano, bem como a forma que a linguagem se relaciona com o aprendizado obtido.

\subsection{Chatbot}

Antes de definir o significado de chatbot, é necessário entender que esses assistentes virtuais inteligentes são um resultado de um avanço em várias áreas da ciência no decorrer dos anos, como a inteligência artificial, processamento de linguagem natural, banco de dados e rede de comunicação de dados (CRUZ; ALENCAR; SCHMITZ, 2018).

A Inteligência Artificial dedicou-se a fazer sistemas similares a humanos, o processamento de linguagem natural, a facilitar a interação do homem com a máquina, banco de dados desenvolveram técnicas de armazenamento e a rede de comunicação com a transferência entre dados e, graças aos avanços dessas áreas, surgiram os assistentes virtuais inteligentes (CRUZ; ALENCAR; SCHMITZ, 2018).

Portanto, para desenvolver um chatbot faz-se necessário a capacidade de entender questões dos seres humanos, estabelecer a heurística, encontrar respostas e consultar banco de dados para compor a resposta de cada pergunta. O estudo da língua e da comunicação também tem se destacado para a evolução dos chatbots, pois a língua é dinâmica e evolui a cada momento, e é importante que as tecnologias estejam atualizadas com o vocabulário atual (CRUZ; ALENCAR; SCHIMTZ, 2018).

Calado (2016) salienta que existem dois tipos de chatbots: com inteligência artificial e baseado em regras. Segundo o mesmo autor, o primeiro caso possui a capacidade de entender 
o que disse, conseguindo aprender e entender a linguagem natural. Já o segundo caso funciona por meio de regras, roteiros prontos ou palavras-chave, ou seja, caso um indivíduo escreva algo diferente do planejado, o chatbot não conseguirá entender e interpretar a interação.

É importante pontuar que chatbots e assistentes virtuais não são iguais, apesar de possuírem características parecidas, existem diferenças entre ambas as tecnologias. Os chatbots interagem por chats, aplicativos de mensagens e são capazes de enviar links e arquivos, de forma automaticamente de acordo com a necessidade do usuário. Já assistentes virtuais utilizam tanto voz quanto textos e suas respostas vão de acordo com o obtido na internet, podendo ser texto, vídeo, imagem entre outras formas. Um exemplo deste caso seria o Google Assistant (MUTANT, 2020).

\begin{tabular}{|c|c|c|}
\hline Ferramenta & Vantaoens & Valores \\
\hline ManyChat & $\begin{array}{l}\text { Permite criar chatbots para suporte ao } \\
\text { cliente, vendas e marketing, podendo } \\
\text { atuar no Facebook Messenger. }\end{array}$ & $\begin{array}{l}\text { Para chatbots pequenos, a versão } \\
\text { gratuita do software é suficiente, mas } \\
\text { para os mais complexos é necessário } \\
\text { adquirir um plano. Inicialmente de } 15 \\
\text { dólares, mas pode subir de acordo com } \\
\text { as interacões. }\end{array}$ \\
\hline Botsify & $\begin{array}{l}\text { Não exige conhecimento de programação } \\
\text { e é possível utilizar o Messenger. Um de } \\
\text { seus clientes em potencial é a Apple.com. } \\
\text { Possui a opção de um transbordo para um } \\
\text { operador humano caso o chatbot não } \\
\text { resolva o problema e gerar relatórios. }\end{array}$ & $\begin{array}{l}\text { Os valores variam. Para negócios } \\
\text { pequenos, pode-se usar uma versão } \\
\text { gratuita, porém, para ter chatbots } \\
\text { ilimitados é preciso uma mensalidade } \\
\text { de } 50 \text { dólares. }\end{array}$ \\
\hline Morph & $\begin{array}{l}\text { Pode ser usado no Facebook e nos sites } \\
\text { particulares da empresa. Não é necessário } \\
\text { conhecimento em programação para } \\
\text { construir o chatbot, mas para quem } \\
\text { possui, poderá incluir seu código no } \\
\text { chatbot. Emite relatórios e possui } \\
\text { configurações especificas. }\end{array}$ & $\begin{array}{l}\text { Comparado com as outras ferramentas } \\
\text { seu valor é mais alto: } 900 \text { dólares } \\
\text { mensais. Por outro lado, oferece mais } \\
\text { recursos além do chatbot, como: } \\
\text { ferramentas de gerenciamento, suporte, } \\
\text { entre outras. }\end{array}$ \\
\hline Chatfuel & $\begin{array}{l}\text { Oferece serviços exclusivos para } \\
\text { empresas, e possui grandes clientes como } \\
\text { os times da NBA e a MTV. Funciona no } \\
\text { Facebook. }\end{array}$ & $\begin{array}{l}\text { É uma ferramenta gratuita, e a empresa } \\
\text { apenas pagará se desejar algo específico } \\
\text { da empresa. }\end{array}$ \\
\hline ChattyPeople & $\begin{array}{l}\text { Oferece serviços para Facebook e Skype, } \\
\text { sem a necessidade de conhecimento em } \\
\text { informática, e outras vantagens como o } \\
\text { machine learning, usando a linguagem } \\
\text { natural. }\end{array}$ & $\begin{array}{l}\text { Possui a versão gratuita limitada e } \\
\text { também planos. }\end{array}$ \\
\hline Sequel & $\begin{array}{l}\text { Pode ser usado em Facebook, Telegram, } \\
\text { Kik e Viber, e possui templates } \\
\text { diferentes. }\end{array}$ & $\begin{array}{l}\text { É possível fazer a criação e o uso } \\
\text { gratuitamente. }\end{array}$ \\
\hline Bluelab & $\begin{array}{l}\text { É uma ferramenta brasileira, e possui } \\
\text { clientes como o Itaú e a Caixa. É capaz de }\end{array}$ & Sem informações sobre os valores. \\
\hline
\end{tabular}




\begin{tabular}{|l|l|l|}
\hline & $\begin{array}{l}\text { entender tanto a linguagem escrita quanto } \\
\text { falada, e oferecem relatórios. }\end{array}$ & \\
\hline Blip & $\begin{array}{l}\text { Também é nacional, oferece relatórios, e } \\
\text { é capaz de interpretar a pergunta do } \\
\text { usuário para responder corretamente. } \\
\begin{array}{l}\text { Pode utilizar os dados já existentes na } \\
\text { empresa e aplicado em Messenger, Skype } \\
\text { ou no site próprio. }\end{array}\end{array}$ & $\begin{array}{l}\text { Para fazer o cadastro na plataforma é } \\
\text { grátis, mas também possui diferentes } \\
\text { planos para as empresas. }\end{array}$ \\
\hline
\end{tabular}

Fonte: Biz2Digital (2020)

Em ambas as ferramentas é necessário desenvolver a linguagem natural e aperfeiçoar a experiência com o usuário, dessa forma, trará vantagens como a diminuição de custos e automatização de processos (MUTANT, 2020).

\subsection{Humanização}

Alan Turing, no ano de 1950, criou um teste a fim de verificar se uma máquina possui uma inteligência satisfatória, que ficou conhecido como Teste de Turing. Ele consiste em fazer perguntas de um humano para o computador, e depois da interação feita por meio de perguntas escritas, o interrogador não conseguirá descobrir se quem respondeu é de fato uma máquina ou outro ser humano (RUSSELL e NORVING, 2013).

Para que o computador seja suficientemente inteligente é necessário que tenha capacidades de se comunicar no idioma natural do ser humano, denominado processamento de linguagem natural, seja capaz de armazenar o que aprendeu e o que aprenderá, ter um raciocínio para buscar as respostas às perguntas feitas e "tirar" suas próprias conclusões, e, por fim, ter aprendizado de máquina, ou seja, ser capaz de se adaptar a circunstâncias e detectar padrões (RUSSELL e NORVING, 2013).

Alan Turing, com seu teste, não apenas criou mais uma teoria sobre máquinas, criou uma ferramenta intelectual, capaz de raciocinar e com o passar do tempo, o próprio computador foi se tornando humanizado, ganhando novas formas e cada vez mais a capacidade de "pensar" (DOMINGUES, 2003).

\subsection{Análise de dados}

A análise de dados contribui para a evolução do chatbot, para que sempre esteja ativo e em produção, conversando sempre com as pessoas que buscarem por eles, e de modo geral, o sucesso dessa tecnologia e uma de suas bases de funcionamento é a análise de dados (WITTEL, 2020). 
Portanto, deve-se entender que o funcionamento do chatbot é simples: ele responderá uma pergunta sem que tenha intervenção humana e tais respostas são formuladas por meio de informações já armazenadas ou um algoritmo que deve ser seguido e, através deles, a própria tecnologia poderá evoluir (WITTEL, 2020).

Segundo Panicali (2019) por isso, fazer uma análise de dados constantemente colabora para o crescimento do chatbot sendo indispensável para medir o desempenho e sua performance. É importante que tudo seja feito de forma automatizada para que não haja latência de tempo lendo cada conversa realizada entre o chatbot e um humano.

Dessa forma, obtendo todos os feedbacks e análises possíveis, observando inclusive, se há abandonos no meio de uma conversa, sendo possível verificar em quais pontos ainda é necessária uma evolução no processo de diálogo (PANICALI, 2019).

\section{ESTUDO DE CASO}

A tecnologia do chatbot voltada para o atendimento ao cliente está sendo implantada na empresa Grupo Oportunidade, a fim de melhorar os procedimentos da central de atendimento.

\subsection{A empresa}

Em 1992 foi fundado a Prodata, que atuava no ramo de cursos e vendas de equipamentos de informática. Um tempo depois, em agosto de 2001 é desenvolvido um método interativo (audiovisual) de ensino, usado inicialmente na empresa Prodata, e com isso, começou o desenvolvimento de cursos. A On byte foi fundada logo depois, em 2002, e a partir de 2010, a empresa iniciou a expansão por franquias.

Logo após, foi criado o Grupo Oportunidade, que engloba todas as marcas e serviços criados pela equipe: Melhor Sistema de Ensino, On byte e Dr. Educa.

Atualmente a Melhor Sistema de Ensino oferece vários cursos profissionalizantes, em um total de aproximadamente 80 cursos de diversas áreas, desde o básico, como digitação, até os mais avançados, como os softwares da Adobe ${ }^{\circledR}$, alcançando cerca de 800 escolas, entre licenças e franquias, espalhadas por todo o país, contando com mais de 150 mil alunos, e também possui uma plataforma totalmente online denominada Dr. Educa.

Além de oferecer a plataforma de cursos interativos, a empresa conta com serviço de consultoria e a central de atendimento, onde os clientes entram em contato para sanar dúvidas ou relatar problemas que possam acontecer. 


\subsection{A decisão}

A empresa já conta com uma central de atendimento preparada para realizar a comunicação com clientes quando estes buscam o suporte necessário, a fim de esclarecer dúvidas, realizar pedidos, registrar sugestões ou, até mesmo, reclamações.

A primeira preocupação e objetivo a ser alcançado é garantir um atendimento ágil, reduzindo o cenário de latência em espera por parte dos clientes, que precisam aguardar para receber a solução de seu problema. E a segunda está relacionada com a afinidade dos usuários finais com o chatbot e com a possibilidade de um desconforto em serem atendidos por uma máquina, e não por um operador humano, fazendo-se, portanto, necessário que o atendimento não seja totalmente robotizado.

Dessa forma, após pesquisas realizadas internamente, analisando diferentes ferramentas e formas de aumentar a excelência no atendimento ao cliente, decidiram utilizar o recurso tecnológico do chatbot, a fim de resolver questões instantaneamente ou caso seja necessário realizar a transferência do atendimento, inicialmente realizado pelo chatbot, para um operador humano, este terá acesso a todas as informações previamente relatadas pelo cliente ao chatbot para agilizar a resolução do problema.

\subsection{Resultados esperados}

O principal resultado esperado era atender os clientes de forma mais rápida, segura e assertiva quando buscassem pela central de atendimento, também de um modo claro e objetivo, resolvendo seus problemas e respondendo suas dúvidas de forma mais eficaz.

Consequentemente, espera-se alcançar um número maior de atendimentos com alto nível de satisfação e resolução de problemas, erradicar a fila de espera de atendimentos ou diminuir em torno de $70 \%$, de forma que os atendentes humanos não fiquem sobrecarregados com problemas que a própria tecnologia do chatbot pode resolver.

Criando uma base de dados maior com todas as dúvidas e os problemas mais frequentes, a fim de evoluir e produzir uma espécie de Frequently Asked Questions - FAQ, para que o próprio chatbot seja capaz de evoluir e ter a capacidade de responder aos clientes.

\subsection{Implantação e funcionamento}

Nos primeiros meses de 2019 foi estudado qual seria a melhor forma de implantar essa tecnologia de chatbots na empresa, fazendo análises e estudos sobre quais seriam os principais 
aspectos e quais os pontos relevantes, sendo colocadas algumas regras iniciais que deveriam ser seguidas. Nesse momento, junto com os operadores da central de atendimento, foi construída a árvore de decisões, demonstrando o "caminho de respostas" que o chatbot deve fazer conforme o cliente vai perguntando, e envia respostas corretas de acordo com as necessidades do usuário.

Já na segunda metade do ano de 2019, nos meses de outubro a dezembro, iniciou-se o processo de desenvolvimento e implantação dessa tecnologia dentro da empresa, toda operação foi realizada por uma empresa terceirizada contratada pelo Grupo Oportunidade, utilizando Javaweb e UX Design (User Experience Design).

Nesse segundo momento, com o auxílio dos atendentes, foram realizados testes e aumentou-se a árvore de decisões, estabelecendo o maior número de respostas possíveis para os clientes, aumentando o número de resoluções de problemas para que o objetivo de diminuir a intervenção de atendentes humanos seja atingido.

Foi observado que vale a Lei de Pareto (economista italiano do século XIX), isto é: $80 \%$ das perguntas e dúvidas dos consumidores tendem a ser respondida por apenas $20 \%$ das respostas providas pelo serviço de atendimento ao cliente (PLUSOFT, 2020).

Esse aspecto possibilita a redução do contato com os operadores da central de atendimento, permite uma otimização dos recursos já existentes e não levariam a um crescimento contínuo no fluxo de atendimento realizado por pessoas, visto que as questões apresentadas possuem um perfil estatístico muito característico.

Tendo sua implantação prevista para ocorrer na primeira metade de 2020 e, uma vez implantado e bem alimentado, o próprio chatbot será capaz de formular respostas e oferecer o melhor atendimento humanizado para todos os consumidores da empresa.

Todos os operadores da central de atendimento foram treinados na utilização da plataforma de atendimento automatizada, incluindo a retirada de relatórios e demais funções básicas dentro do próprio chatbot.

Os clientes têm acesso ao chatbot por vários canais de comunicação, incluindo site próprio da empresa e o atendimento por troca de mensagens através do Whatsapp, mas independentemente do modo escolhido para suporte, o atendimento será o mesmo. Além desses meios já em funcionamento, pretende-se incluir o Facebook, Facebook Messenger e até mesmo o Instagram para realizar esses atendimentos. 
A linguagem utilizada dentro do chatbot foi toda embasada nos melhores conceitos de experiência do usuário, seguindo a tradição já incorporada nos processos da empresa desde o início no uso hotline e helpdesk, para que eles tivessem prazer ao fazer acesso à plataforma e ser atendido pela equipe, que inclui o chatbot.

De forma simplificada, o usuário que já possui um cadastro realiza o login no site ou faz a solicitação do atendimento por um número de telefone cadastrado em bancos de dados internos, é atendido de início pelo chatbot, que recupera as informações básicas desse cliente com base em atendimentos antigos e em seu cadastro, como problemas anteriores, sua cidade, se possui boletos em atraso ou não, entre outras informações.

Assim que a interação com o chatbot for iniciado, o usuário poderá fazer perguntas que serão respondidas automaticamente, baseadas em informações já obtidas ou inseridas na própria base de dados da ferramenta.

Caso o problema ou requisição necessite de uma intervenção humana, o atendimento será transferido para um operador da central de atendimento que já terá em mãos todo o histórico da conversa, portanto, saberá quem é o cliente, qual o problema ou a dúvida pendente, consequentemente, agilizando a resolução.

\section{RESULTADOS E DISCUSSÃO}

A princípio não foi uma tarefa simples fazer com que os clientes optassem pelo atendimento por um "robô" e não por um atendente humano real. Mas, com o passar do tempo perceberam que a plataforma também é eficiente e pode resolver problemas, da mesma forma que um atendente humano faria.

Pode-se dizer que a implantação do chatbot na empresa Grupo Oportunidade foi apropriada e, inicialmente diminuiu cerca de $30 \%$ de atendimentos realizados por operadores ou com qualquer tipo de intervenção humana, segundo averiguado pelo sistema de atendimento, em comparação da quantidade de atendimentos feitos antes e após a implantação do chatbot, ou seja, alguns clientes já passaram apenas a utilizar o chatbot para esclarecer suas dúvidas ou realizar algum pedido.

O tempo exato de atendimento não é possível ser calculado pelo fato de os atendimentos serem de assuntos variados, alguns mais rápidos que os outros. Mas pode-se dizer que, mensalmente, antes da implantação do chatbot, eram realizados aproximadamente 5000 atendimentos por operadores humanos. Com o chatbot, esse número caiu 30\%, ou seja, 1500 
inserções foram atendidas apenas pelo chatbot, sem a necessidade da intervenção de um atendente, de acordo com dados apurados pelo sistema de atendimento.

Ainda existem melhorias que podem ser feitas para alcançar o resultado almejado, que é diminuir os atendimentos para operadores humanos em torno de 70\%. Para isso, ainda é necessário incentivar os clientes a utilizarem a plataforma, provando que o chatbot não é apenas uma máquina, mas também trabalha de forma humanizada, sendo capaz de compreender e responder de forma correta.

É oportuno observar que também será realizada uma análise dos dados para identificar quais pontos ainda precisam de melhorias, atualizações e evoluções, e qual etapa do atendimento está funcionando corretamente.

A inteligência artificial colaborará durante todo o processo, visto que está presente em todo momento do desenvolvimento do chatbot, e graças a ela, a tecnologia dos atendimentos automatizados está evoluindo, fazendo com que o objetivo da empresa seja alcançado.

Quadro 2 - A implantação do chatbot - antes e após

\begin{tabular}{|l|l|}
\hline \multicolumn{1}{|c|}{ Antes da implantação do chatbot } & \multicolumn{1}{c|}{ Após a implantação do chatbot } \\
\hline $\begin{array}{l}\text { Os atendimentos eram feitos apenas pelo chat da } \\
\text { empresa. }\end{array}$ & $\begin{array}{l}\text { Os atendimentos passaram a ser feitos por vários } \\
\text { canais de comunicação, incluindo o chat, } \\
\text { Whatsapp e Facebook Messenger. }\end{array}$ \\
\hline $\begin{array}{l}\text { A central de atendimento funcionava das 8h às } \\
\text { 20h de segunda à sexta, e aos sábados das 8h às } \\
16 \mathrm{~h}\end{array}$ & $\begin{array}{l}\text { Os operadores humanos continuam atuando no } \\
\text { mesmo período, porém o chatbot atua durante } 24 \\
\text { horas para sanar dúvidas. }\end{array}$ \\
\hline $\begin{array}{l}\text { Havia um tempo maior de espera na fila de } \\
\text { atendimentos, já que um atendente conversava } \\
\text { com até } 4 \text { pessoas. }\end{array}$ & $\begin{array}{l}\text { A espera reduziu no primeiro momento, visto que } \\
\text { alguns problemas passaram a resolvidos com o } \\
\text { atendimento no chatbot. }\end{array}$ \\
\hline $\begin{array}{l}\text { Atendimentos simples, como a solicitação de de } \\
\text { instaladores ou respostas poderia demorar, em } \\
\text { média, de 20 minutos a 1 hora. }\end{array}$ & $\begin{array}{l}\text { Atendimentos passaram a ter, em média de 10 a } \\
\text { 20 minutos de duração. O cliente faz a solicitação } \\
\text { e, imediatamente, recebe um link com o que } \\
\text { necessita. }\end{array}$ \\
\hline $\begin{array}{l}\text { Problemas mais complicados, com relação a a } \\
\text { banco de dados corrompidos, por exemplo, } \\
\text { demoravam em torno de } 3 \text { horas, desde a a } \\
\text { verificação inicial até a resolução do problema. }\end{array}$ & $\begin{array}{l}\text { Os problemas mais complicados passaram a ser } \\
\text { resolvidos em 90 minutos. O operador humano } \\
\text { apenas intervirá na conversa após todas as } \\
\text { verificações terem sido feitas e descoberto o } \\
\text { problema maior. O operador apenas precisa } \\
\text { solucionar essa questão. }\end{array}$ \\
\hline $\begin{array}{l}\text { Um cliente que retornava com um problema não } \\
\text { solucionado, gerava uma latência em recuperar } \\
\text { histórico atendimento, a fim de verificar quais } \\
\text { soluções já foram aplicadas, demorando ainda } \\
\text { mais a resolução do problema. }\end{array}$ & $\begin{array}{l}\text { Como o cliente possui um cadastro no sistema, } \\
\text { com seu e-mail e telefone cadastrado, ao entrar } \\
\text { em contato com o chatbot, automaticamente todo } \\
\text { o histórico de atendimento é apresentando, } \\
\text { facilitando para o operador a visualização do } \\
\text { problema e as tentativas de correção feitas. }\end{array}$ \\
\hline
\end{tabular}

Fonte: Autoria própria (2020) 


\section{CONSIDERAÇÕES FINAIS}

O desenvolvimento do estudo representou mais uma oportunidade e cenário em um mundo está cada vez mais inserido na era de tecnologias inteligentes e as empresas também estão se atualizando e inovando em suas de formas de trabalhar, procurando oferecer os melhores produtos e as melhores soluções para seus clientes.

Dessa forma, implantar um chatbot voltado ao atendimento ao cliente não só resolve problemas ou responde dúvidas que pessoas levam até ele, mas também traz uma excelência ao atendimento, garante um equilíbrio na sobrecarga de chamadas e as torna mais ágil e eficiente, de forma que gera e manipula um histórico e demais dados para possíveis buscas.

É importante ter em mente que um atendimento excelente ao cliente e ao usuário é um diferencial competitivo para as empresas que buscam uma posição relevante no mercado altamente globalizado e que opera em uma rotina de 24 por 7 ao longo da semana de trabalho.

Por fim, o trabalho apresentado mostrou que a inovação no atendimento a clientes com a intermediação de uma ferramenta inteligente e, atuando de forma humanizada, o chatbot não permitiu apenas melhorar procedimentos simples, como responder às perguntas, mas também trouxe racionalidade ao mecanismo de retorno aos clientes e usuários dos produtos e serviços oferecidos, alcançando uma excelência no atendimento.

\section{REFERÊNCIAS}

BIZ2DIGITAL. Chatbot: as melhores ferramentas do mercado. Disponível em: $<$ https://biz2digital.com.br/chatbot-as-melhores-ferramentas-do-mercado/>. Acesso em: 17 abr. 2020.

CALADO, Caio. Afinal, o que é chatbot e como ele pode melhorar o atendimento nas empresas? 23 de abril de 2016. Disponível em <https://take.net/blog/chatbots/o-que-echatbot/>. Acesso em 23 mar. 2020.

CRUZ, Leôncio Teixeira; ALENCAR, Antonio Juarez; SCHMITZ, Eber Assis. Assistentes Virtuais Inteligentes e Chatbots: Um guia prático e teórico sobre como criar experiências e recordações encantadoras para os clientes da sua empresa. Rio de Janeiro, Brasil. BRASPORT Livros e Multimídia Ltda, 2018.

DOMINGUES, Diana. A Arte no século XXI: A humanização das tecnologias. 1.ed. 4. Reimpressão. Brasil. 2003. 
HAYKIN, Simon. Redes Neurais: Princípios e prática. Porto Alegre, Brasil. Bookman, 1999. LUGER, George F. Artificial Intelligence: Structures and Strategies for Complex Problem Solving. 6. ed. Boston: Person, 2009.

MUTANT. Você sabe qual a diferença entre chatbot e assistente virtual? Disponível em: $<$ https://mutantbr.com/blog/diferenca-entre-chatbot-e-assistente-virtual/>. Acesso em 11 abr. 2020.

PANICALI, Ana Paula. Análise de dados para evolução de chatbots. 18 de abril de 2019 . Disponível em: <https://take.net/blog/chatbots/analise-de-dados-para-evolucao-de-chatbots $>$. Acesso em 25 mar. 2020.

PLUSOFT. Assistentes Virtuais Inteligentes Excelência e Redução de Custo Operacional. ABRIL/ 2014. https://www.plusoft.com.br/wp-content/uploads/2015/06/ whitepaper-AVI.pdf. Acesso em 11 abr. 2020.

ROSA, João Luís Garcia. Fundamentos da Inteligência Artificial. Rio de Janeiro, Brasil: Gen | LTC, 2011.

RUSSELL, Stuart; NORVING, Peter. Inteligência Artificial. 3. ed. [S. 1.]: Elsevier, 2013.

SHAWAR, Bayan Abu, ATWELL, Eric. Chatbots: Are they Really Useful? 2007.

WITTEL. Chatbots: como a inteligência artificial vem mudando o serviço ao cliente. Disponível em: <https://blog.wittel.com/chatbot-servico-cliente/>. Acesso em: 26 mar. 2020. 


\section{DECLARAÇÃO DE AUTORIZAÇÃO}

Autorizamos para os devidos fins, a senhora Verônica Adelaide Lugli, R.G 56.799.941-5, a divulgar o nome, os dados e as fotos da empresa Grupo Oportunidade, CNPJ 24.445753/0001-79, em seu Trabalho de Conclusão de Curso: 0 uso do chatbot para a excelência em atendimento, a ser apresentado na Faculdade de Tecnologia de Taquaritinga/SP.

A aluna compromete-se a não utilizar/divulgar, por nenhum meio, os dados confidenciais da referida empresa.

Taquaritinga, 07 de abril de 2020.

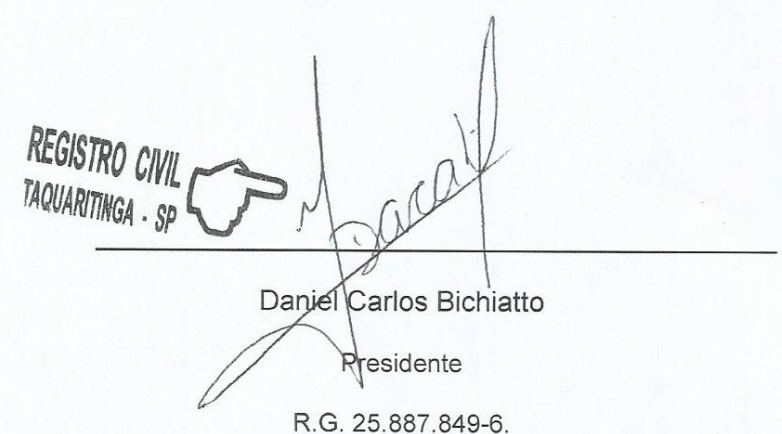

R.G. $25 \cdot 887.849-6$ 